\title{
Executive functioning in schizophrenia and the relationship with symptom profile and chronicity
}

\author{
KATHRYN E. GREENWOOD, ${ }^{1}$ ROBIN MORRIS, ${ }^{1}$ THORDUR SIGMUNDSSON, ${ }^{2}$ \\ SABINE LANDAU, ${ }^{3}$ AND TIL WYKES ${ }^{1}$ \\ ${ }^{1}$ Department of Psychology, Institute of Psychiatry, London, United Kingdom \\ ${ }^{2}$ Department of Psychiatry, Landspitalinn, The University Hospital, Reykjavik, Iceland \\ ${ }^{3}$ Department of Biostatistics and Computing, Institute of Psychiatry, London, United Kingdom \\ (Received January 22, 2008; Final Revision June 20, 2008; Accepted June 20, 2008)
}

\begin{abstract}
This study reports the executive function profile in people with schizophrenia, with a simultaneous comparison of chronicity and of those with predominately disorganization versus psychomotor poverty symptoms. The patients were split into one set defined according to symptoms (29 with disorganization, 29 with negative symptoms) and the other representing chronicity (22 first-episode, 35 chronic) and compared with 28 healthy controls on a broad range of executive process measures. Differences were investigated in both the severity and profile of impairments. Impairment patterns interacted with symptom groups, with disorganization and psychomotor poverty symptom groups showing different profiles of executive impairment. In contrast, across these same executive processes, impairment profiles were similar between first episode and chronic schizophrenia and became more similar, particularly for working memory, when controlling for disorganization symptoms. The executive profile, therefore, is related to symptom type rather than chronicity. (JINS, 2008, 14, 782-792.)
\end{abstract}

Keywords: Schizophrenia, Psychosis, Neuropsychology, Executive function, Symptoms, Chronic

\section{INTRODUCTION}

The term "executive function" is used to cover a variety of fractionated cognitive processes concerned with the control, organization and sequencing of higher cognition. This umbrella term includes such functions as working memory, initiation, response inhibition, problem solving, and planning (Burgess \& Simons, 2005). Studies have estimated that $27-46 \%$ of people with schizophrenia have selective "executive" profiles and 54-90\% have at least one executive impairment (Chan et al., 2006a,b; Johnson-Selfridge \& Zalewski, 2001; Kremen et al., 2004). Executive dysfunction is associated with poor social outcome (Kopelowicz et al., 2005; Laes \& Sponheim, 2006) and has become the target for neurorehabilitation (Wykes et al., 2007a). The impairment has been related neurobiologically to the connectivity between the prefrontal cortex and the striatal cir-

Correspondence and reprint requests to: Kathryn E. Greenwood, Department of Psychology, Institute of Psychiatry, De Crespigny Park, London SE5 8AF, UK. E-mail: k.greenwood@iop.kcl.ac.uk cuitry, forming the substrate for organization processes that control cognition (Elliott, 2003).

Several factors may impact on the characteristics of executive dysfunction in schizophrenia. A main consideration is the relationship with symptoms, linked both theoretically and empirically with the pattern of neuropsychological impairment (Hill et al., 2002; Kremen et al., 2004; Liddle, 1987a,b). Models of schizophrenia have suggested a distinct syndromic structure (Emsley et al., 2003; Liddle, 1987a,b; Mass et al., 2000) such as a demarcation into three syndromes, namely reality distortion, disorganization, and psychomotor poverty (negative symptoms). The latter two of these are associated with executive dysfunction (See Donohoe \& Robertson, 2003), as in the theory by Frith (1992): Disorganization symptoms arise from impaired inhibition of habitual responses when plans must be constructed and implemented using working memory, whereas psychomotor poverty results from deficits in the initiation of activities due to impaired initiation of plans. In studies of single symptoms, both syndromes have been associated with impaired verbal initiation and working memory and disor- 
ganization also with attention, inhibition, discourse planning and monitoring (Hoffman et al., 1986; Liddle \& Morris, 1991; Pantelis et al., 2001). Although the symptom structures have been debated, all recent refinements of symptom models retain the distinction between disorganization and psychomotor poverty. Previous studies have compared profiles across domains in relation to symptoms, or have provided fine-grained analyses within executive functions (Chan et al., 2006a,b; Hill et al., 2002; Hutton et al., 1998; Kremen et al., 2004; Liddle \& Morris, 1991), but there has been no direct comparison of profiles on a broad range of executive processes in relation to both disorganization and psychomotor poverty. Due to the over-reliance on a "unitary view of executive function in schizophrenia", theoretical accounts that dissociate executive processes, such as Frith's theory, have remained largely unexplored (Donohoe \& Robertson, 2003).

A second consideration is chronicity, which cuts across symptom distinctions. To study this issue, researchers have compared functioning in first episode and chronic schizophrenia across different domains. First episode schizophrenia shows executive dysfunction at this early stage, with some degree of clinical heterogeneity (Chan et al., 2006b; Joyce et al., 2005; Joyce \& Roiser, 2007), but less impairment than is found in chronic schizophrenia (Chan et al., 2006a; Saykin et al., 1994). Profiles have varied between studies, with parallel flat profiles of diffuse general impairment, parallel nonflat profiles with selective impairments, and selective impairments specific to chronic schizophrenia (Albus et al., 1996; Blanchard \& Neale, 1994; Chan et al., 2006a,b; Saykin et al., 1994). These variations might result from studies that collapse test scores across broad domains. This is problematic for executive functions that fractionate into uncorrelated processes (Burgess \& Simons, 2005). Nevertheless, the main pattern appears to be that profiles are broadly the same in the two stages of the illness, suggesting a core pattern of dysfunction.

Executive profiles are less clearly linked than memory and globally impaired profiles to symptom subgroups, perhaps due to the unitary approach, but are associated with cognitive and symptom severity and illness duration (Chan et al., 2006a,b; Hill et al., 2002; Kremen et al., 2004). Categorization of subgroups by neuropsychological profile in all cases confounds the relationship between symptoms and chronicity. Hence the current study explores the severity and profile of executive functioning in relation to disorganization and psychomotor poverty. Simultaneously, it investigates the distinction between first episode and chronic schizophrenia. An overlapping sampling method is used in which a general pool of people with schizophrenia are split either by symptoms or according to chronicity, hence the simultaneous comparisons. Furthermore, the study determines whether symptom groupings differentiate on the basis of a fine-grained analysis of executive function and whether the early and late profiles are similar. Four main aspects of executive functioning are explored: working memory, planning and strategy formation, response initiation, and response inhibition. Based on theoretical accounts of schizophrenia that consider fractionated executive function (e.g., Frith, 1992), disorganization was predicted to be associated with a broader range of executive deficit whilst psychomotor poverty would be associated with impairments in working memory and response initiation. The study explores whether the overall pattern of impairment remains when comparing first episode versus chronic patients.

\section{METHOD}

\section{Design}

Executive functioning was compared in a cross-sectional sample of people with schizophrenia and healthy controls, the former split by symptoms or chronicity for two parallel analyses. The healthy control group provided a comparison for group effects and a normative reference for profile analyses. General intellectual functioning was controlled.

\section{Participants}

All participants were sampled from the same inner-city geographical area and were aged $18-65$, with a screen IQ estimate over 70, using the National Adult Reading TestRestandardized (Nelson \& Willison, 1991), English as a first language, no neurological problems or head injury, and no current substance abuse. The 28 control participants were screened by self-report on past mental health problems and contact with psychiatric services and were recruited provided they had no psychiatric history.

The schizophrenia participants were hospitalized or were outpatients and satisfied DSM-IV criteria confirmed using: DSM-IV checklist, clinical notes and consultant psychiatrist collateral information. People with schizoaffective disorder were excluded. Symptoms were assessed using SAPS and SANS (Andreassen, 1984a,b). Disorganization was defined as scoring at least 8 from SAPS formal thought disorder and inappropriate affect and SANS poverty of content of speech items. Psychomotor Poverty was defined as having at least one core symptom of alogia or flat affect (summed score at least 6) and one other negative symptom (summed score at least 3 on any remaining SANS subscale) (Liddle, 1987a). First episode participants comprised all eligible participants with schizophrenia recruited as part of a large population-based study of first-episode psychosis (Dazzan et al., 2004). DSM-IV diagnoses were additionally confirmed through consensus and 6-month follow-up. Participants were identified within 1 week of their first psychiatric contact for psychosis and were assessed as soon as their symptoms had stabilized (see Table 1). Chronic illness was defined as at least 5 years from first psychiatric contact for psychosis, beyond any period of initial deterioration. Chronicity was verified by self-report and the supporting collateral clinical information. 
Table 1. Sociodemographic and clinical characteristics for the symptom sample

\begin{tabular}{|c|c|c|c|c|c|c|c|c|c|}
\hline \multirow[b]{3}{*}{ Age (years) } & \multirow{2}{*}{\multicolumn{2}{|c|}{$\begin{array}{l}\text { Controls } \\
(n=28)\end{array}$}} & \multirow{2}{*}{\multicolumn{2}{|c|}{$\begin{array}{l}\text { Psychomotor poverty } \\
\qquad(n=29)\end{array}$}} & \multirow{2}{*}{\multicolumn{2}{|c|}{$\begin{array}{l}\text { Disorganization } \\
\quad(n=29)\end{array}$}} & \multicolumn{3}{|c|}{ Statistical test } \\
\hline & & & & & & & $\begin{array}{c}\text { Test } \\
\text { statistic }\end{array}$ & $d f$ & $p$ \\
\hline & 33.1 & $(7.34)$ & 33.9 & $(8.81)$ & 36.2 & $(8.04)$ & $F=1.2$ & 2,83 & .31 \\
\hline Sex (\%male $)$ & \multicolumn{2}{|c|}{89} & \multicolumn{2}{|c|}{93} & \multicolumn{2}{|c|}{86} & $\chi^{2}=0.74$ & 2 & .69 \\
\hline Parental SES & \multicolumn{2}{|c|}{$3 / 15 / 10$} & \multicolumn{2}{|c|}{$4 / 13 / 9(n=7)$} & \multicolumn{2}{|c|}{$2 / 18 / 8(n=8)$} & $\chi^{2}=1.4$ & 4 & .48 \\
\hline Education(yrs) & 14.5 & $(2.81)$ & 12.8 & $(2.37)$ & 12.1 & $(2.19)$ & $F=7.0$ & 2,83 & .002 \\
\hline Premorbid IQ & 110.0 & $(6.54)$ & 97.0 & $(12.1)$ & 96.8 & $(11.5)$ & $F=14.8$ & 2,83 & .001 \\
\hline Current IQ & 113.2 & $(16.0)$ & 90.0 & $(17.6)$ & 88.7 & $(12.7)$ & $F=22.2$ & 2,83 & .001 \\
\hline Illness Length (Yrs) & & & 7.83 & $(7.4)$ & 11.9 & $(8.55)$ & $F=3.9$ & 1,56 & .053 \\
\hline Medication dose & & & 48.3 & $(27.2)$ & 49.9 & $(39.6)$ & $F=0.03$ & 1,56 & .86 \\
\hline$\%$ on atypicals & & & \multicolumn{2}{|c|}{$62 \%$} & \multicolumn{2}{|c|}{$50 \%$} & $\chi^{2}=0.81$ & 1 & .37 \\
\hline Reality distortion & & & 14.7 & $(12.0)$ & 16.1 & $(9.25)$ & $F=0.27$ & 1,56 & .60 \\
\hline Disorganization & & & 1.79 & $(2.15)$ & 14.8 & $(4.98)$ & & $*$ & \\
\hline Psychomotor poverty & & & 19.6 & $(6.66)$ & 8.26 & $(6.30)$ & & $*$ & \\
\hline General negative & & & 15.8 & $(6.7)$ & 18.1 & $(6.13)$ & $F=1.9$ & 1,56 & .17 \\
\hline
\end{tabular}

Note. Mean (Standard deviation) unless otherwise stated. Medication dose was converted to a standard total percentage of the maximum recommended dose (Maudsley and Bethlem Prescribing Guidelines 1996). Parental SES was divided into 3 categories $1=$ professional $/$ managerial, $2=$ skilled, $3=$ semi-skilled/unskilled. * Different due to group definition.

The study was reviewed and ethical committee approval was obtained. After a description of the study, all the participants gave written informed consent.

A total of 71 participants were allocated to either or both samples according to fulfillment of membership criteria. The chronicity sample comprised 22 first episode and 35 chronic participants, while the symptom sample encompassed 29 designated as disorganization and 29 as psychomotor poverty. Some participants fulfilled the criteria for only one sample because disorganization/psychomotor poverty symptoms were absent or too mild to fulfill symptom criteria $(n=13)$ or because illness length lay between first episode and chronic sample criteria $(n=14)$.

\section{Measures}

\section{Socioeconomic status}

Parental socioeconomic status was derived from self-report of paternal occupation at participant's birth, which was categorized into one of five broadly defined categories from 1 (professional) to 5 (unskilled) (Office of Population Consensus' and Surveys, 1991).

\section{General intellectual function}

Intellectual function was assessed with the NARTRestandardized and current IQ with a recognized WAIS-R short form (Canavan et al., 1986).

\section{Executive function}

Tests were selected to measure a range of well defined primary executive processes known to be affected in schizophrenia, including working memory, planning/strategy use, response initiation, and response inhibition, measured across verbal and spatial domains (Baddeley, 2000).

\section{Working memory}

The tasks were chosen to distinguish between verbal and spatial maintenance and manipulation processes (Baddeley, 2000; D’Esposito et al., 1999).

For verbal working memory the digit span forward test was used to measure maintenance (Baddeley, 2000), with span defined as the longest sequence recalled correctly on $2 / 3$ trials. Manipulation was measured using the Verbal Working Memory Word Span task, a simplified version of the Daneman and Carpenter (1980) sentence span task. The task requires remembering words, with interleaving decision making concerning recognition of previously presented words and relies on maintenance with additional manipulation requirements (Greenwood, 2000). Single word lists were presented consecutively on a computer screen, starting with two words and increasing by one word every four sequences. After list presentation, recognition memory for the probe word was tested with selection of a "yes" or a "no" box on the screen. The probe was a target word on $50 \%$ of occasions, a word closely associated with the target on $25 \%$ and a distracter on $25 \%$ of occasions. Immediately after selection, the participants were required to recall the word list. The manipulation measure was the longest sequence of words (word span) recalled correctly on at least 3 of 4 trials.

For Spatial Working Memory, the Executive Golf Task, a version of the Spatial Working Memory task (SWT) (Hutton et al., 1998; Joyce et al., 2005; Owen et al., 1996) was used that encompasses the distinction between maintenance and manipulation. This is a naturalistic version of the 
task with three dimensional graphics (Feigenbaum et al., 1996; Miotto et al., 1996), which has been used in schizophrenia (Kravariti et al., 2007; Toulopoulou et al., 2003, 2004). This task (see previous papers for description) involves a series of searches for hidden targets on a computer screen, with nonreturn to the successful target on subsequent searches. A maintenance measure is derived from errors in returning to the same location during a single search, whilst a measure of manipulation is based on errors that occur when the participant returns to a previously successful location between searches. Studies have shown that within search errors dissociate from between search errors in patients with focal temporal and frontal lesions (Feigenbaum et al., 1996; Miotto et al., 1996).

\section{Planning}

This was measured with the 3D-Computerised Tower of London task (Kravariti et al., 2003, 2007; Morris et al., 1995), which is based on the Shallice Tower of London task (1982) and is similar to the Stockings of Cambridge computerized task (CANTAB: Robbins et al., 1994). The task involves moving discs around a display in a certain sequence, using a touch sensitive screen, to achieve a target arrangement. The main score used in this study is the Mean Moves above Minimum required to solve 4 or 5 move problems. This measure, used in previous studies with similar procedures is sensitive to frontal lobe damage affecting planning ability (e.g., Owen et al., 1990).

\section{Strategy use}

Verbal strategy formation was assessed using a modified version of the Hayling task (Burgess \& Shallice, 1996). The original task required the completion of sentences with a single meaningless completion word and was modified to include sentences with both high and low dominance prepotent responses. Common strategies are to look for items in the room (visual) or to link successive responses semantically (verbal). A total strategy scoring method is based on the procedure by Burgess and Shallice (1996). Spatial strategy use was assessed using a measure of strategy formation on the Executive Golf Task. More efficient search paths involved sampling proximal locations in a sequential strategic manner. A higher score constituted poorer strategy use. This measure shows selective strategy formation deficits in patients with frontal lobe lesions (Owen et al., 1996) and dissociates strategy formation and working memory deficits in schizophrenia (e.g., Pantelis et al., 2001).

\section{Response initiation}

This was measured using a modified verbal fluency task which alternated between searches by letter (FAS version) and by category (Animals, Fruits, Body Parts) with 30-second trials for each (Greenwood, 2000; Spreen \& Benton, 1977).

\section{Response inhibition}

This was estimated verbally by taking the number of incorrect meaningful completion words produced on the modified Hayling task. This response inhibition estimate has been shown through factor analysis to be distinct from the estimate of strategy use derived using the same test (Burgess \& Shallice, 1996). Spatially, response inhibition was assessed using the incompatible minus the compatible response latency on the Complex Reaction Time (4-button) task, which has been used previously as a measure of response inhibition (Wykes et al., 1990).

\section{Medication dose}

To obtain a single medication dose across typical and atypical neuroleptics, dose was converted to a standard total percentage of the maximum recommended dose using the Maudsley and Bethlem Prescribing Guidelines (1996).

\section{Statistical Analyses}

\section{Assessment of group differences in level of function}

Group differences in executive functioning were investigated using MANCOVAs of the executive variables. WAIS-R IQ was covaried to control for the relationship between schizophrenia and general cognitive impairment, and for the relationship between tests due to cognitive congruence. This increased the specificity to explore the relationship between schizophrenia and distinct executive functions. The analysis allowed for IQ to have a different effect on each of the measures under investigation. The assumed linear relationship between executive functions and IQ within each group was assessed using residual plots and no evidence of nonlinearity was found. The linear effect of IQ did not vary between groups in the respective interaction tests, which were not statistically significant at the 5\% level. Age and education were not controlled as the former covaries with chronicity and the latter is disrupted as a side effect of the onset of schizophrenia. Post hoc pairwise group comparisons were carried out on group differences with multistage Bonferroni corrections to control for multiple group comparisons.

\section{Assessment of differences within executive profiles}

Executive functioning profiles were investigated by transforming executive variables to $Z$-scores by reference to the normal controls and then analyzing the effect of the withinsubject factor, executive process, and its interaction with the between-subject factor, group. Profile analyses were conducted on the schizophrenia groups using generalized estimating equations (GEE) to fit linear-models for the $Z$-scores that assumed normally distributed errors with constant variances (Hardin, 2005). The model allowed for correlations 
between the repeated $Z$-scores by assuming an exchangeable covariance structure. To account for a more complex covariance structure and nonconstant errors, the robust sandwich estimator was used (Williams, 2000).

For each profile analysis, the interaction between group and executive process was investigated. If this was significant it was maintained in the model and further investigations were carried out for each group separately. Otherwise the main effect of executive process was assessed across groups. An additional interaction term between executive process and IQ was fitted.

\section{Assessment of specific islets of strength or deficit}

Four subdomains were derived to reflect those identified by Shallice and Burgess (1996). Planning and strategy use were considered part of the New Schema Construction subdomain. Response inhibition was incorporated within the Assessment and Verification of Schema subdomain. The third subdomain, Implementation of Schema within Working Memory, was considered to incorporate both working memory and response initiation which are hypothesized to be differentially associated with different symptoms and so were separated. Each domain score (planning/strategy use, response inhibition, working memory, response initiation) was compared with the average of all other process scores while holding IQ constant at 100. When the GEE analysis identified an interaction between group and executive process, the process comparisons were conducted within each group separately, otherwise comparisons were carried out across groups. The multi-stage Bonferroni procedure was used to control for multiple domain or group by domain comparisons.

\section{Assessment of the impact of symptoms} on the chronicity analysis

As was expected due to sampling factors in our crosssectional design, symptoms were associated with chronicity. Specifically, disorganization symptoms were greater in the chronic group (see Table 2). As this might affect chronicity profiles, a GEE analysis of the chronicity sample controlled for more severe forms of disorganization using a new severe thought disorder factor which was entered into the analysis. The criterion for severe thought disorder was a score of 4 or more on at least one of the SAPS thought disorder measures. Ten participants with chronic and two with first episode schizophrenia fulfilled criteria for high thought disorder severity. No control was made for psychomotor poverty which did not differ between chronicity groups.

\section{Assessment of the impact of medication on executive function.}

GEE analyses of medication dose [high (greater than 50\% of the maximum dose) compared with low dose] and group (standard neuroleptics, clozapine, and other atypicals) assessed medication effects. In each case, standardized scores were derived using one group (Low dose or Standard Neuroleptics respectively) as the reference.

All analyses were conducted using STATA (Statistical Data Analysis) version 7.0.

Table 2. Sociodemographic and clinical characteristics for the chronicity study

\begin{tabular}{|c|c|c|c|c|c|c|c|c|c|}
\hline \multirow[b]{3}{*}{ Age (years) } & \multirow{2}{*}{\multicolumn{2}{|c|}{$\begin{array}{l}\text { Controls } \\
(n=28)\end{array}$}} & \multirow{2}{*}{\multicolumn{2}{|c|}{$\begin{array}{l}\text { First Episode } \\
\quad(n=22)\end{array}$}} & \multirow{2}{*}{\multicolumn{2}{|c|}{$\begin{array}{l}\text { Chronic } \\
(n=35)\end{array}$}} & \multicolumn{3}{|c|}{ Statistical test } \\
\hline & & & & & & & $\begin{array}{c}\text { Test } \\
\text { statistic }\end{array}$ & $d f$ & $p$ \\
\hline & 33.1 & $(7.34)$ & 28.6 & $(9.9)$ & 38.1 & $(6.9)$ & & $*$ & \\
\hline Sex (\%male) & \multicolumn{2}{|c|}{89} & \multicolumn{2}{|c|}{82} & \multicolumn{2}{|c|}{94} & $\chi^{2}=2.2$ & 2 & .33 \\
\hline Parental SES & \multicolumn{2}{|c|}{$3 / 15 / 10$} & $1 / 12 / 8$ & $(n=21)$ & $4 / 19 / 11$ & $(n=5)$ & $\chi^{2}=0.87$ & 4 & .93 \\
\hline Education(yrs) & 14.5 & $(2.81)$ & 13.1 & $(2.98)$ & 12.6 & $(2.25)$ & $F=4.1$ & 2,82 & .02 \\
\hline Premorbid IQ & 110.0 & $(6.54)$ & 93.7 & $(8.9)$ & 99.5 & $(12.4)$ & $F=17.8$ & 2,82 & .001 \\
\hline Current IQ & 113.2 & $(16.0)$ & 92.2 & $(17.2)$ & 91.1 & (14.1) & $F=18.3$ & 2,82 & .001 \\
\hline Illness length (yrs) & & & 0.18 & $(0.20)$ & 14.6 & $(6.7)$ & & $*$ & \\
\hline Medication dose & & & 41.6 & $(42.4)$ & 52.5 & (31.3) & $F=1.1$ & 1,54 & .29 \\
\hline$\%$ on atypicals & & & \multicolumn{2}{|c|}{$50 \%$} & \multicolumn{2}{|c|}{$42 \%$} & $\chi^{2}=0.37$ & 1 & .57 \\
\hline Reality distortion & & & 19.2 & (9.19) & 16.9 & $(9.17)$ & $F=0.78$ & 1,55 & .38 \\
\hline Disorganization & & & 4.5 & $(5.9)$ & 10.6 & (7.6) & $F=10.5$ & 1,55 & .002 \\
\hline Psychomotor poverty & & & 7.8 & $(7.9)$ & 11.6 & $(8.7)$ & $F=2.9$ & 1,55 & .095 \\
\hline General negative & & & 11.5 & $(7.5)$ & 18.7 & $(4.7)$ & $F=19.7$ & 1,55 & .001 \\
\hline
\end{tabular}

Note. Medication dose was converted to a standard total percentage of the maximum recommended dose (Maudsley and Bethlem Prescribing Guidelines 1996). Parental SES was grouped into 3 categories $1=$ professional/managerial, $2=$ skilled, $3=$ semi-skilled/unskilled. * Different due to group definition. 


\section{RESULTS}

\section{Demographics}

The groups were balanced for sex and parental socioeconomic status, which is purportedly the optimal demographic matching variable for schizophrenia (Keefe, 1995; see Tables 1 and 2). The first episode group was, by design, younger than the chronic sample, but the difference is in an age range where substantive age-related cognitive differences are not expected. All schizophrenia groups had significantly fewer years of education and lower IQ than controls. The IQ of the control sample did not differ significantly from their demographically predicted premorbid IQ. This IQ estimate is largely determined by social class which was matched between the schizophrenia and control samples (Crawford \& Allan, 1997). The control sample, therefore, was estimated to perform at the level that the schizophrenia sample might otherwise have attained, based on demographics, had they not developed schizophrenia. There were no education or IQ differences between any of the schizophrenia groups. The schizophrenia groups were well balanced on clinical characteristics, except for the expected symptom differences. The chronic group demonstrated statistically greater disorganization and general negative symptoms (asociality, anhedonia, avolition, and apathy) compared with first episode schizophrenia. They were also well-balanced for medication use with no statistically significant differences between groups in neuroleptic dose or the proportion of participants who were taking atypical neuroleptics.

\section{Symptoms Sample}

\section{Group differences in level of function}

Figure 1 shows the executive profiles. A significant group effect was detected after controlling for IQ [Wilks lambda $=$ $0.36, F(20,128)=4.21 ; p<.001]$. Post hoc pairwise between group comparisons revealed poorer performance in each schizophrenia group compared with controls [Wilks lambda $=0.32, F(10,39)=8.13 ; p<.001$ for psychomotor poverty and Wilks lambda $=0.55, F(10,41)=3.29 ; p=$ .003 for disorganization] and a trend for poorer performance in the disorganization compared with the psychomotor poverty syndrome [Wilks lambda $=0.67, F(10,38)=$ $1.89 ; p=.07$ compared with $\alpha=.05 / 1]$.

\section{Differences within executive profiles}

The profile comparisons revealed a significant interaction between executive process and group $\left[\chi^{2}(9)=25.01 ; p=\right.$ .003] after adjusting for the interaction between executive process and IQ $\left[\chi^{2}(9)=24.85 ; p=.003\right]$. Post hoc and selectivity analyses revealed significant within-group effects of executive process in both the psychomotor poverty and the disorganization groups $\left[\chi^{2}(9)=96.9 ; p<.0001 ; \chi^{2}(9)=\right.$ 55.6; $p<.0001]$ consistent with different executive profiles in these groups.

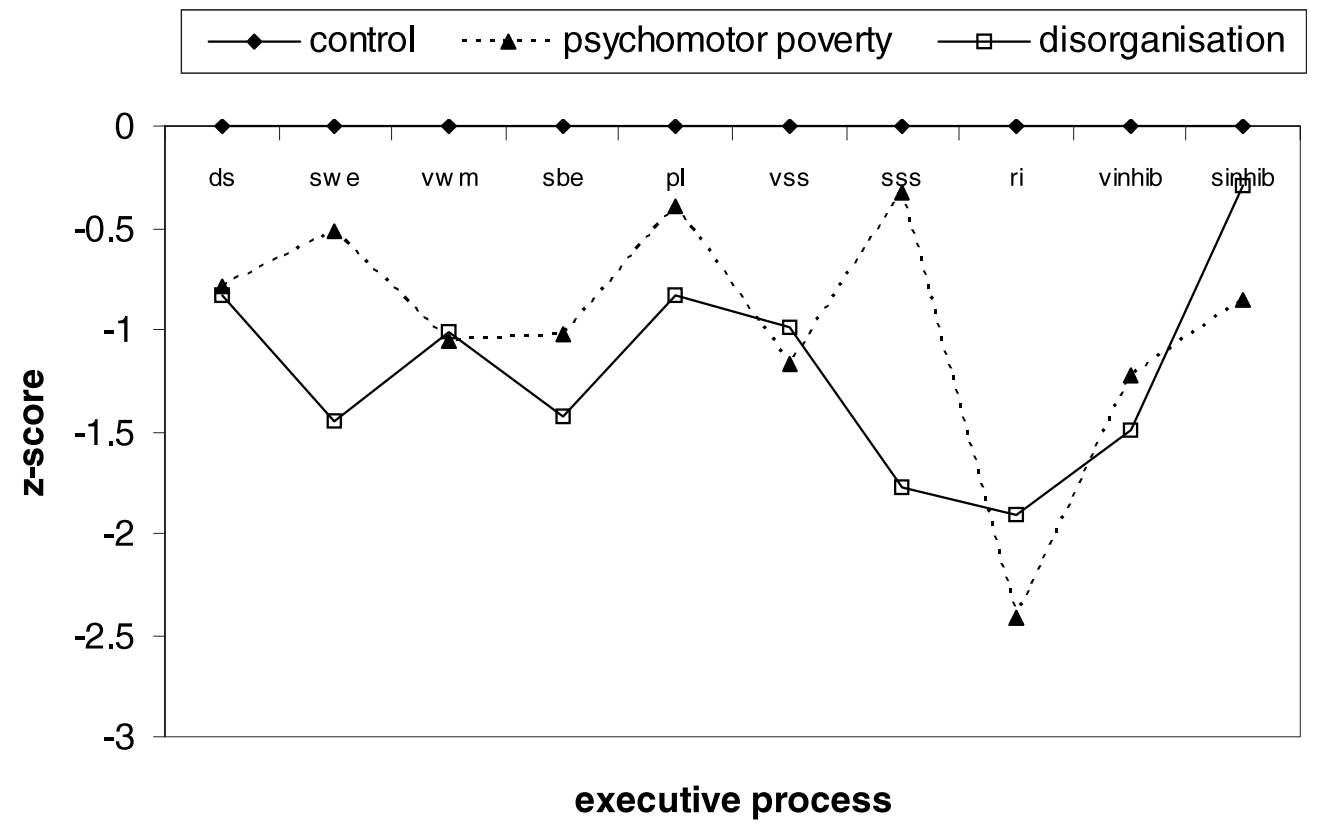

Fig. 1. Executive Impairment Profiles in the Psychomotor Poverty $(n=29)$ and Disorganization syndromes $(n=29)$ of schizophrenia compared with normal control performance $(n=28)$ and with current IQ held constant at 100. Working Memory $[\mathrm{ds}=$ digit span, $\mathrm{swe}=$ spatial within search errors, vwm $=$ verbal working memory, $\mathrm{sbe}=$ spatial between search errors]; Planning and Strategy $[\mathrm{pl}=$ planning, vss = verbal strategy score, sss = spatial strategy score]; Response Initiation-Inhibition [ri = response initiation, vinhib = verbal inhibition, sinhib = spatial inhibition]. 
Assessment of specific islets of strength or deficit

The analyses revealed a selective responds initiation impairment within both the psychomotor poverty and disorganization groups compared with the average of all other processes [(estimated difference in mean $Z$-scores $=-1.61$; $Z=-8.14 ; p<.001 ; \mathrm{CI}(95)=(-1.99$ to -0.22$)$ for Psychomotor Poverty and $=-0.79 ; Z=-2.67 ; p=.007$, $\mathrm{CI}(95)=(-1.36$ to -0.21$)$ for Disorganization $)$ compared, according to the multistage Bonferroni procedure, with $\alpha=$ $.05 / 7$ and .05/6, respectively]. Within the Psychomotor Poverty group, planning and strategy use was relatively intact compared with the average of all other processes [estimated difference in mean $Z$-scores $=0.50 ; Z=3.51 ; p<.0001$; $\mathrm{CI}(95)=(0.22-0.77)$ compared with $\alpha=.05 / 8]$. However, Figure 1 suggests that this relative strength was determined by planning and spatial strategy use.

\section{Chronicity Comparison}

\section{Group differences in level of function}

Lower scores were observed in the chronic compared with the first episode group on all but one measure (Figure 2). A significant group effect was found after controlling for IQ [Wilks lambda $=0.50 ; F(20,130)=2.5 ; p<.001]$. Post hoc pair-wise between-group comparisons revealed poorer performance in chronic schizophrenia [Wilks lambda $=0.48$; $F(10,47)=5.01 ; p<.001]$ and a trend for poorer performance in first episode schizophrenia compared with con- trols [Wilks lambda $=0.59 ; F(10,34)=2.39 ; p=.029$ compared with $\alpha=.05 / 2$ ] but no statistical difference between chronicity groups [Wilks lambda $=0.71 ; F(10,39)=$ $1.59 ; p=.15]$. As there was no group difference between first episode and chronic schizophrenia, the impact of age on the group main effect was not investigated further.

\section{Differences within executive profiles}

There was no interaction between group and executive process $\left[\chi^{2}(9)=14.5 ; p=.106\right]$, but there was a significant effect of executive process $\left[\chi^{2}(9)=55.7 ; p<.0001\right]$ and a significant interaction between executive process and IQ $\left[\chi^{2}(9)=35.9 ; p<.0001\right]$, consistent with parallel nonflat executive profiles in first episode and chronic schizophrenia. The two chronicity groups were, therefore, combined and the remaining post hoc and selectivity analyses were conducted within-groups.

\section{Assessment of specific islets of strength or deficit}

The analyses revealed a selective impairment in response initiation compared with each other process [(estimated difference in mean $Z$-scores $=-1.03 ; Z=-5.62 ; p<.001$, $\mathrm{CI}(95)=(-1.39$ to -0.67$)$ compared, according to the multistage Bonferroni procedure, to $\alpha=.05 / 4$ )].

\section{Assessment of the impact of controlling} for symptoms on the chronicity analysis

Disorganization symptoms but not psychomotor poverty symptoms differed between the two chronicity groups. It was predicted that the executive profiles would, therefore,

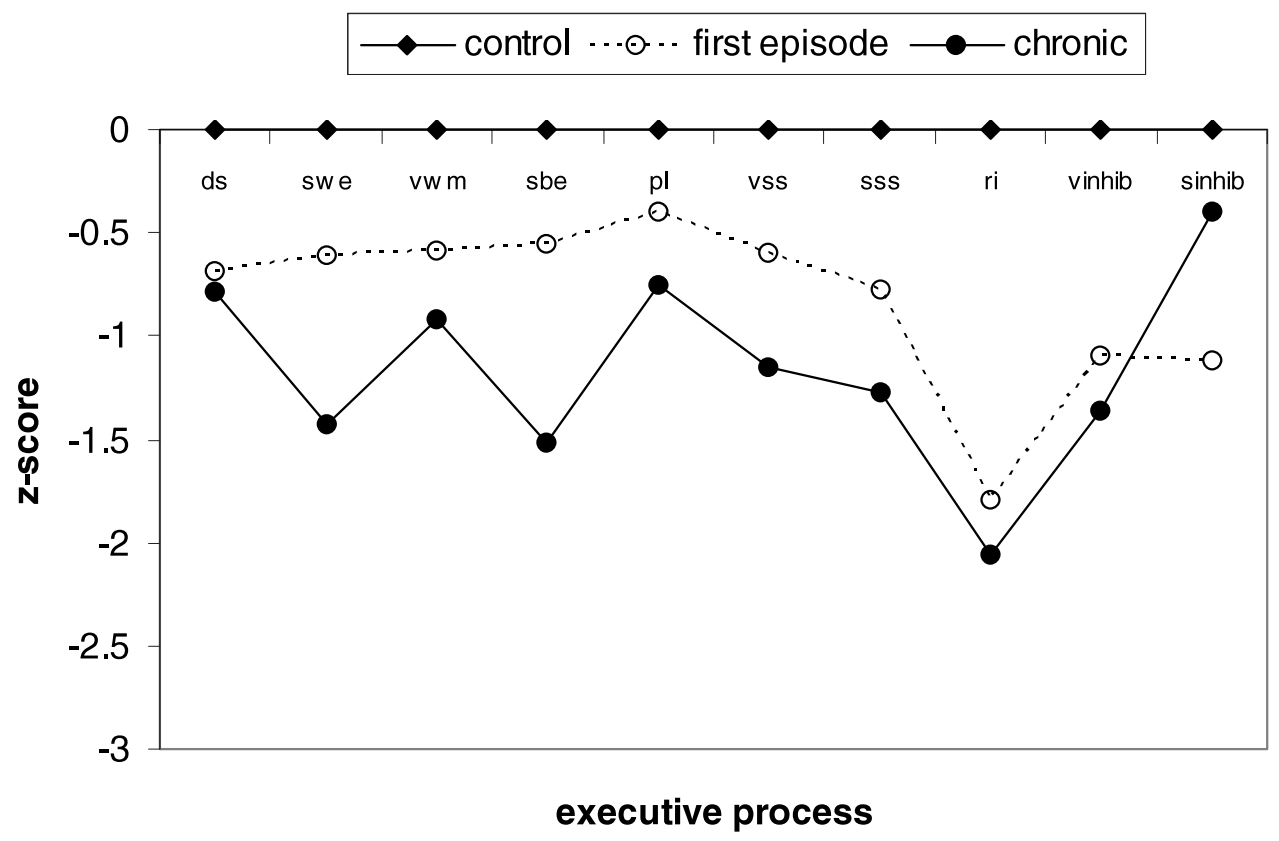

Fig. 2. Executive Impairment Profiles in First episode $(n=22)$ and Chronic Schizophrenia $(n=35)$ compared with normal control performance $(n=28)$ and with current IQ held constant at 100 (see Figure 1 for measure labels). 
be more similar in first episode and chronic schizophrenia if the distribution of disorganization symptoms was controlled. The results for the level of impairment analysis remained unchanged with a significant group effect [Wilks lambda $=0.53 ; F(20,128)=2.40 ; p=.002$ ], a post hoc between-group difference in chronic schizophrenia [Wilks lambda $=0.50 ; F(10,46)=4.6 ; p<.001]$ and a trend for a difference in first episode schizophrenia compared with healthy controls [Wilks lambda $=0.55 ; F(10,33)=2.25$; $p=.039$ compared with $\alpha=.05 / 2$ ] but no difference between chronicity groups $[$ Wilks lambda $=0.73 ; F(10,38)=1.42$; $p=.21]$.

The profile analysis also remained unchanged with a significant effect of executive process $\left[\chi^{2}(9)=51.2 ; p<\right.$ $.0001]$ so that even after adjusting for disorganization symptoms the within-group profiles were significantly nonflat.

The analysis of impairments, after controlling for disorganization, again indicated a significant response initiation impairment [estimated difference between domain and average of remaining domains $=-1.08 ; Z=-5.53 ; p<.001$; $\mathrm{CI}(95)=(-1.47$ to -0.70$)]$ but now also indicated relatively intact working memory (estimated difference $=0.32$; $Z=2.45 ; p=.014$ compared with $\alpha=.05 / 3 ; \mathrm{CI}(95)=$ (.065-0.58). A comparison between Figures 2 and 3 revealed that this reflected the more similar profiles in first episode and chronic schizophrenia, particularly in working memory, after controlling for disorganization.

\section{Assessment of the impact of medication on executive function}

There were no significant main effects of either medication dose or group on executive function $(p$ 's $>.10)$.

\section{DISCUSSION}

The study demonstrated fractionated profiles of executive impairments, after controlling for IQ, which differed in relation to symptom type but not in relation to chronicity. The differential pattern of executive impairments in schizophrenia is thus determined by symptoms and not chronicity. Executive impairments in the disorganization group were generally greater and encompassed a broader range of processes than in the psychomotor poverty group. In the disorganization group, planning/strategy use was as impaired as the majority of other processes while in the psychomotor poverty group it was more intact than other processes. When disorganization was controlled, profiles were closer and working memory was statistically more intact across first episode and chronic schizophrenia, which suggests an association between disorganization and working memory impairments. Response initiation was selectively impaired to different degrees in all schizophrenia groups but this impairment was greatest in the psychomotor poverty group. The results support the original theoretical proposition by Frith (1992) that negative symptoms may be underpinned by impaired initiation of novel responses while disorganization symptoms incorporate a broader range of impairments including inhibition and planning. This pattern of multiple selective deficits within executive function is consistent with predicted differential fractionation of executive function in different symptom groups (Chan et al., 2006a) and with patterns described previously across different cognitive domains in schizophrenia as a whole (Sullivan et al., 1994).

In contrast, consistent with some but not all studies, profiles were parallel and did not differ statistically in severity between first episode and chronic schizophrenia. Most stud-

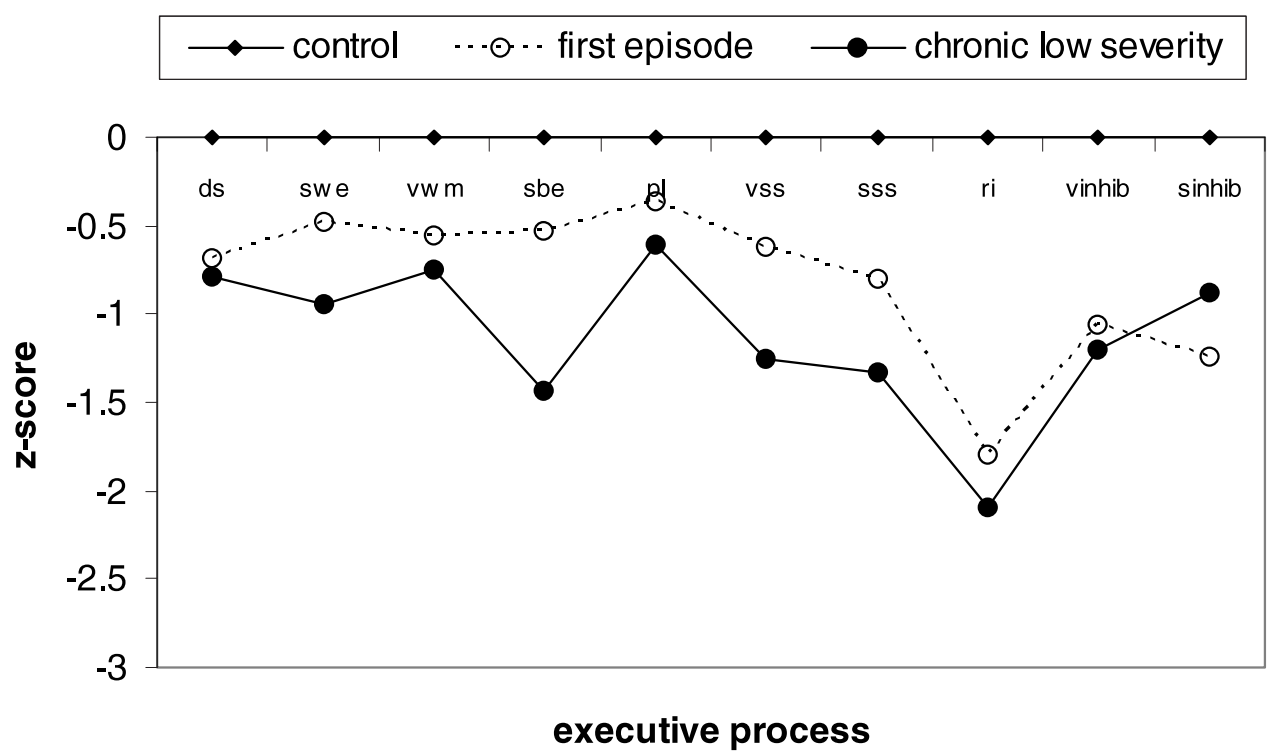

Fig. 3. Executive Impairment Profiles in First Episode Schizophrenia and Chronic Schizophrenia with lower disorganization severity compared with normal control performance and with current IQ held constant at 100. See Figure 1 for measure labels. 
ies focused on cognition more broadly rather than on executive processes (Censits et al., 1997; Saykin et al., 1994). Some studies showed significantly greater executive impairment in chronic compared with first episode schizophrenia (Albus et al., 1996). Others showed a wide range of executive impairments at first episode but a disproportionate number (46\%) with intact executive function (Chan et al., 2006b). Here, the chronic group performed more poorly than the first episode sample on 9 of 10 measures so the lack of statistical significance may have reflected some lack of power.

This research is novel in demonstrating in a single study that schizophrenia as a whole, whether first episode or chronic, appears to be characterized by a single executive profile. Yet, this executive profile seems likely to reflect the symptom make-up of the samples. The similar impairment pattern in first episode and chronic samples suggests a similar but attenuated proportional mix of different symptomspecific executive impairment profiles in first episode compared with chronic schizophrenia. The less severe impairment profile in first episode schizophrenia may have reflected the intact executive performance in those with first episode schizophrenia who will not become chronic. This attenuated executive impairment in first episode schizophrenia may either result from a proportion of the first episode sample with flat, intact profiles similar to controls or from a characteristic executive profile throughout the sample which nevertheless lies above the control mean and in the intact range for a proportion of the first episode sample. This intact cognition group may be those with good outcomes.

Poor cognition is a recognized marker of poor outcome in schizophrenia from first episode and appears to contribute to this outcome independently of symptoms (Dickinson $\&$ Coursey, 2002; Moritz et al., 2000). There are difficulties in identifying stable symptoms that are predictive of poor outcome at first episode (Fujii \& Wylie, 2003; Malla et al., 2004) and the predictive power of executive functions on first episode outcome appears short-lived (Goldstein et al., 2002). This may reflect the tendency for executive functions, negative and disorganization symptoms to improve (Schuepbach et al., 2002) with improved prospective outcome. The combined stable presence of these executive impairments and symptoms at first episode may together indicate poor outcome. The targeted remediation of these candidate "chronicity" markers may prove beneficial in remediating the poor outcome in these symptom groups (Wykes et al., 2007a,b).

This study has several limitations. A single balanced sample across chronicity and symptoms would have allowed the measurement of their independent effects and interactions on profiles. However, even in the current large sample, few first-episode participants met symptom criteria so the power of the study to detect unadjusted group differences would be compromised. The measures were selected to encompass a comprehensive range of executive processes within four broad domains that are commonly impaired in schizophrenia, but some processes were not tested directly. The individual measures were selected to most closely reflect a single individual process and general cognition was controlled but several processes may still have contributed to performance on each individual measure (Jaeger et al., 2003). Most schizophrenia participants were taking neuroleptic medication, but there were no differences in dose or medication type between schizophrenia groups. Chronicity did not interact with executive functioning, making it unlikely that life-time medication exposure, which would covary with chronicity, mediated differences in executive functioning. This is consistent with findings that executive dysfunction is present in first episode neuroleptic naïve schizophrenia and persists after controlling for medication use in chronic samples (Chan et al., 2006a,b).

This study addresses issues identified in previous investigations of executive function in schizophrenia (Chan \& Toulopoulou, 2006; Donohoe \& Robertson, 2003). The approach to executive function is theory-driven and incorporates the fractionation of executive functions. This specificity of executive process impairment from general impairment is further enhanced through the covariation of current intellectual function and of component executive processes. Symptom subgroups and illness duration were investigated simultaneously to separate their impact on quantitative (severity) and qualitative executive impairments in schizophrenia (Kremen et al., 2004). The study includes a sizeable disorganization group, and the recruitment of a first episode sample early in their first presentation minimizes the impact of long-term medication effects and onset associated cognitive decline.

The fractionation of executive functions precludes a single executive profile as might occur for other functional domains. However, the current limitations in our theoretical and empirical knowledge of executive function in schizophrenia tend to prevent more detailed subtyping by fractionated executive processes. An alternative approach, specifically when considering executive function in schizophrenia, is to consider subgroups defined by symptoms which are then investigated for their differential executive profiles. The refinements in the understanding of symptom subtypes which now go well beyond early positive-negative dichotomies, yet still retain the negative and disorganization distinction, combined with a greater understanding of vulnerability factors linked to symptom subtypes, provides greater empirical support for a symptom approach. These symptom distinctions appear reliable across diagnostic boundaries and in their associations with executive impairments (Kravariti et al., 2005). The merits of this approach lie in the advanced understanding of the relationship between executive function and the phenomenology of schizophrenia, with predicted advances in understanding and remediating chronic schizophrenia with poor functional outcome.

\section{ACKNOWLEDGMENTS}

This study was supported by a PhD studentship from the Medical Research Council. We thank Professors Robin Murray and Julian 
Leff, Institute of Psychiatry, for enabling collaboration with the AESOP project to recruit people with first episode psychosis and for providing consensus diagnoses in this group.

\section{REFERENCES}

Albus, M., Hubmann, W., Ehrenberg, C., Forcht, U., Mohr, F., Sobizack, N., Walheim, C., \& Hecht, S. (1996). Neuropsychological impairments in first episode and chronic schizophrenia patients. European Archives of Psychiatry and Clinical Neuroscience, 246, 249-255.

Andreassen, N. (1984a). SANS: Scale for the Assessment of Negative Symptoms. Iowa City, IA: University of Iowa.

Andreassen, N. (1984b). SAPS: Scale for the Assessment of Positive Symptoms. Iowa City, IA: University of Iowa.

Baddeley, A.D. (2000). Short-term and working memory. In E. Tulving \& F.I.M. Craik (Eds.), The Oxford Handbook of Memory (pp. 77-92). New York: Oxford University Press.

Blanchard, J. \& Neale, J. (1994). The neuropsychological signature of schizophrenia: Generalised or differential deficit? American Journal of Psychiatry, 151, 40-48.

Burgess, P.W. \& Shallice, T. (1996). Response suppression, initiation and strategy use following frontal lobe lesions. Neuropsychologia, 34, 263-272.

Burgess, P.W. \& Simons, J.S. (2005). Theories of frontal lobe executive function: Clinical applications. In P.W. Halligan \& D.T. Wade (Eds.), Effectiveness of Rehabilitation for Cognitive Deficits (pp. 211-231). New York: Oxford University Press.

Canavan, A., Dunn, G., \& McMillan, T.M. (1986). Principal components of the WAIS-R. British Journal of Clinical Psychology, 25, 81-85.

Censits, D., Ragland, D., Gur, R., \& Gur, R. (1997). Neuropsychological evidence supporting a neurodevelopmental model of schizophrenia: A longitudinal study. Schizophrenia Research, 24, 289-298.

Chan, R.C.K., Chen, E.Y.H., Cheung, E.F.C., Chen, R.Y.K., \& Cheung, H.K. (2006a). The executive components of executive functioning in a cohort of patients with chronic schizophrenia: A multiple single-case study design. Schizophrenia Research, 81, 173-189.

Chan, R.C.K., Chen, E.Y.H., \& Law, C.W. (2006b). Specific executive dysfunction in patients with first-episode medicationnaive schizophrenia. Schizophrenia Research, 82, 51-64.

Chan, R.C.K. \& Toulopoulou, T. (2006). Fractionation of executive function in schizophrenia: Relationships to clinical and neurological manifestations. In D.P. French (Ed.), Schizophrenia Psychology: New Research (pp. 1-39). Hauppauge, New York: Nova Science Publishers, Inc.

Crawford, J.R. \& Allan, K.M. (1997). Estimating premorbid IQ with demographic variables: Regression equations derived from a U.K. sample. The Clinical Neuropsychologist, 11, 192-197.

Daneman, M. \& Carpenter, P. (1980). Individual differences in working memory and reading. Journal of Verbal Learning and Verbal Behaviour, 19, 450-466.

Dazzan, P., Morgan, K., Orr, K.G., Hutchinson, G., Chitnis, X., Suckling, J., Fearon, P., Salvo, J., McGuire, P.K., Mallett, R.M., Jones, P.B., Leff, J., \& Murray, R.M. (2004). The structural brain correlates of neurological soft signs in AESOP firstepisode psychoses study. Brain, 127, 143-153.

D'Esposito, M., Postle, B.R., Ballard, D., \& Lease, J. (1999). Maintenance versus manipulation of information held in working memory: An event-related fMRI study. Brain and Cognition, 41, 66-86.

Dickinson, D. \& Coursey, R.D. (2002). Independence and overlap among neurocognitive correlates of community functioning in schizophrenia. Schizophrenia Research, 56, 161-170.

Donohoe, G. \& Robertson, I.H. (2003). Can specific deficits in executive functioning explain the negative symptoms of schizophrenia? A review. Neurocase, 9, 97-108.

Elliott, R. (2003). Executive functions and their disorders. British Medical Bulletin, 65, 49-59.

Emsley, R., Rabinowitz, J., \& Torreman, M. (2003). RIS-INT-35 Early Psychosis Global Working Group. The factor structure for the Positive and Negative Syndrome Scale (PANSS) in recent-onset psychosis. Schizophrenia Research, 61, 47-57.

Feigenbaum, J.D., Polkey, C.E., \& Morris, R.G. (1996). Deficits in spatial working memory after unilateral temporal lobectomy in man. Neuropsychologia, 34, 163-176.

Frith, C. (1992). The Cognitive Neuropsychology of Schizophrenia. Hove, UK: Laurence Erlbaum.

Fujii, D.E. \& Wylie, A.M. (2003). Neurocognition and community outcome in schizophrenia: Long-term predictive validity. Schizophrenia Research, 59, 219-223.

Goldstein, R.Z., Giovannetti, T., Schullery, M., Zuffante, P.A., Lieberman, J.A., Robinson, D.G., Barr, W.B., \& Bilder, R.M. (2002). Neurocognitive correlates of response to treatment in formal thought disorder in patients with first-episode schizophrenia. Neuropsychiatry, Neuropsychology and Behavioural Neurology, 15, 88-98.

Greenwood, K.E. (2000). The Nature and Stability of Executive Impairments in Schizophrenia. Ph.D. Thesis: London, England University of London.

Hardin, J.W. (2005). Generalised estimating equations. In B.S. Everitt \& D.C. Howell (Eds.), Encyclopedia of Statistics in Behavioural Science. Chichester, UK: Wiley.

Hill, S.K., Ragland, J.D., Gur, R.C., \& Gur, R.E. (2002). Neuropsychological profiles delineate distinct profiles of schizophrenia, an interaction between memory and executive function, and uneven distribution of clinical subtypes. Journal of Clinical and Experimental Neuropsychology, 24, 765-780.

Hoffman, R., Stopek, S., \& Andreassen, N. (1986). A comparative study of manic vs. schizophrenic speech disorganization. Archives of General Psychiatry, 43, 831-838.

Hutton, S., Puri, B., Duncan, L., Robbins, T., Barnes, T., \& Joyce, E. (1998). Executive function in first episode schizophrenia. Psychological Medicine, 28, 463-473.

Jaeger, J., Czobor, P., \& Berns, S.M. (2003). Basic neuropsychological dimensions in schizophrenia. Schizophrenia Research, $65,105-116$.

Johnson-Selfridge, M. \& Zalewski, C. (2001). Moderator variables of executive functioning in schizophrenia: Meta-analytic findings. Schizophrenia Bulletin, 27, 305-316.

Joyce, E.M., Hutton, S.B., Mutsatsa, S.H., \& Barnes, T.R. (2005). Cognitive heterogeneity in first-episode schizophrenia. British Journal of Psychiatry, 187, 516-522.

Joyce, E.M. \& Roiser, J.P. (2007). Cognitive heterogeneity in schizophrenia. Current Opinion in Psychiatry, 20, 268-272.

Keefe, R. (1995). The contribution of neuropsychology to psychiatry. American Journal of Psychiatry, 152, 6-15.

Kopelowicz, A., Liberman, R.P., Ventura, J., Zarate, R., \& Mintz, J. (2005). Neurocognitive correlates of recovery from schizophrenia. Psychological Medicine, 35, 1165-1173. 
Kravariti, E., Dixon, T., Frith, C., Murray, R., \& McGuire, P. (2005). Association of symptoms and executive function in schizophrenia and bipolar disorder. Schizophrenia Research, 74, 221-231.

Kravariti, E., Morris, R.G., Rabe-Hesketh, S., Murray, R.M., \& Frangou, S. (2003). The Maudsley early onset schizophrenia study: Cognitive function in adolescents with recent onset schizophrenia. Schizophrenia Research, 61, 137-148.

Kravariti, E., Morris, R.G., Rabe-Hesketh, S., Murray, R., \& Frangou, S. (2007). Comparative profile analysis of cognitive function in recent-onset and chronic patients with adolescentonset schizophrenia. Schizophrenia Research, 94, 240-244.

Kremen, W.S., Seidman, L.J., Faraone, S.V., Toomey, R., \& Tsuang, M.T. (2004). Heterogeneity of schizophrenia: A study of individual neuropsychological profiles. Schizophrenia Research, 71, 307-321.

Laes, J.R. \& Sponheim, S.R. (2006). Does cognition predict community function only in schizophrenia?: A study of schizophrenia patients, bipolar affective disorder patients, and community control subjects. Schizophrenia Research, 84, 121-131.

Liddle, P. (1987a). The symptoms of chronic schizophrenia: A re-examination of the positive-negative dichotomy. British Journal of Psychiatry, 151, 145-151.

Liddle, P. (1987b). Schizophrenic syndromes, cognitive performance and neurological dysfunction. Psychological Medicine, 17, 49-57.

Liddle, P. \& Morris, D. (1991). Schizophrenic syndromes and frontal lobe performance. British Journal of Psychiatry, 158, 340-345.

Malla, A.K., Norman, R.M., Takhar, J., Manchanda, R., Townsend, L., Scholten, D., \& Haricharan, R. (2004). Can patients at risk for persistent negative symptoms be identified during their first episode of psychosis? Journal of Nervous and Mental Diseases, 192, 455-463.

Mass, R., Schoemig, T., Hitschfeld, K., Wall, E., \& Haasen, C. (2000). Psychopathological syndromes of schizophrenia: Evaluation of the dimensional structure of the positive and negative syndrome scale. Schizophrenia Bulletin, 26, 167-177.

Miotto, E., Bullock, P., Polkey, C., \& Morris, R.G. (1996). Spatial working memory and strategy formation in patients with frontal lobe excisions. Cortex, 32, 613-630.

Moritz, S., Krausz, M., Gottwalz, E., Lambert, M., Perro, C., Ganzer, S., \& Naber, D. (2000). Cognitive dysfunction at baseline predicts symptomatic 1-year outcome in first-episode schizophrenics. Psychopathology, 33, 48-51.

Morris, R.G., Rushe, T., Woodruffe, P.W.R., \& Murray, R.M. (1995). Problem solving in schizophrenia: A specific deficit in planning ability. Schizophrenia Research, 14, 235-246.

Nelson, H. \& Willison, J. (1991). NART: National Adult Reading Test (NART): Test Manual Second Edition). Windsor, Berkshire, UK: NFER.

Office of Population Consensus' and Surveys. (1991). Standard Occupational Classifications: Vol. 1-3. London: HMSO.

Owen, A., Downes, J.J., Sahakian, B.J., Polkey, C.E., \& Robbins, T.W. (1990). Planning and spatial working memory following frontal lobe lesions in man. Neuropsychologia, 28, 1021-1034.

Owen, A.M., Morris, R.G., Sahakian, B.J., Polkey, C.E., \& Robbins, T.W. (1996). Double dissociations of memory and exec- utive functions in working memory tasks following frontal lobe excisions, temporal lobe excisions or amygdalo hippocampectomy in man. Brain, 119, 1597-1615.

Pantelis, C., Stuart, G.W., Nelson, H.E., Robbins, T.W., \& Barnes, T.R. (2001). Spatial working memory deficits in schizophrenia: Relationship with tardive dyskinesia and negative symptoms. American Journal of Psychiatry, 158, 1276-1285.

Robbins, T.W., James, M., Owen, A., Sahakian, B.J., McInnes, L., \& Rabbitt, P. (1994). Cambridge Neuropsychological Test Automated Battery (CANTAB): A factor analytic study of a large sample of normal elderly volunteers. Dementia, 5, 266-281.

Saykin, A., Shtasel, D., Gur, R., Kester, D., Mozley, L., Stafiniak, P., \& Gur, R. (1994). Neuropsychological deficits in neurolepticnaive patients with first episode schizophrenia. Archives of General Psychiatry, 51, 124-131.

Schuepbach, D., Keshavan, M.S., Kmiec, J.A., \& Sweeney, J.A. (2002). Negative symptom resolution and improvements in specific cognitive deficits after acute treatment in first-episode schizophrenia. Schizophrenia Research, 53, 249-261.

Shallice, T. (1982). Specific impairments in planning. Philosophical Transactions of the Royal Society of London. Series B, Biological Sciences, 298, 199-209.

Shallice, T. \& Burgess, P. (1996). The domain of supervisory processes and temporal organization of behaviour. Philosophical Transactions of the Royal Society of London. Series B, Biological Sciences, 351, 1405-1411.

Spreen, O. \& Benton, A. (1977). Neurosensory Centre for the Comprehensive Examination of Aphasia (NCCEA) Revised Edition. Victoria, Canada: University of Victoria, Neuropsychological Laboratory.

Sullivan, E.V., Shear, P.K., Zipursky, R.B., Sagar, H.J., \& Pfefferbaum, A. (1994). A deficit profile of executive, memory, and motor functions in schizophrenia. Biological Psychiatry, 36, 641-653.

Toulopoulou, T., Grech, A., Schulze, K., McDonald, C., Morris, R.G., Rabe-Hesketh, S., \& Murray, R.M. (2004). Volumetric brain regions and their relation to cognitive function: A family study on schizophrenia. Biological Psychiatry, 56, 447-453

Toulopoulou, T., Morris, R.G., Rabe-Hesketh, S., \& Murray, R.M. (2003). Selectivity of verbal memory deficit in schizophrenic patients and their relatives. American Journal of Medical Genetics. Part B, Neuropsychiatric Genetics, 116, 1-7.

Williams, R.L. (2000). A note on robust variance estimations for cluster-correlated data. Biometrics, 56, 645-646.

Wykes, T., Newton, E., Landau, S., Rice, C., Thompson, N., \& Frangou, S. (2007b). Cognitive remediation therapy (CRT) for young early onset patients with schizophrenia: An exploratory randomized controlled trial. Schizophrenia Research, 94, 221-230

Wykes, T., Reeder, C., Landau, S., Everitt, B., Knapp, M., Patel, A., \& Romeo, R. (2007a). Cognitive remediation therapy in schizophrenia: Randomised controlled trial. British Journal of Psychiatry, 190, 421-427.

Wykes, T., Sturt, E., \& Katz, R. (1990). The prediction of rehabilitative success after three years: The use of social, symptom and cognitive variables. British Journal of Psychiatry, 157, 865-870. 